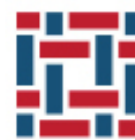 Current Trends in Fashion Technology \& Textile Engineering ISSN: 2577-2929}

\title{
Textile Industry - An Overview
}

\section{Koushik CV and Prakash C*}

Department of Fashion Technology, Sona College of Technology, Salem, India

Submission: January 09, 2018; Published: February 13, 2018

*Corresponding author : Prakash C, Department of Fashion Technology, Sona College of Technology, Salem, India, Tel: +91 98422 66095;

Email: dearcprakash@gmail.com

\begin{abstract}
This paper is an attempt to provide fundamental information on the textile industry, covering broadly the areas of fibre, yarn and fabric production. It provides a crisp outline of common textile fibres and steps involved in the conversion of fibre to yarn, yarn to fabric and fabric wet processing for value addition. Besides students of the textile discipline, this article would also be useful to intermediate level employees engaged in diferent aspects of textile manufacture. Consumers and home economists looking for guidance in the selection and care of textile products might also find it interesting.
\end{abstract}

Keywords: Fibres; Yarn; Filament; Weaving; Knitting; Dyeing; Printing; Finishing

\section{Introduction}

The textile industry primarily comprises the production of yarn, fabric and finished goods. Textile manufacturers procure or produce raw fibres and transform them into yarn, thread or webbing; they convert the yarn into fabric; they colour the yarn or fabric and finish them to have special attributes; finally they use the fabric to construct a variety of end-use products [1].

The basic raw material used in textile manufacturing is the textile fibre, which consists of two types: natural fibres and manufactured (or man-made) fibres (Figure 1).

A classification of textile fibres is shown in Table 1.

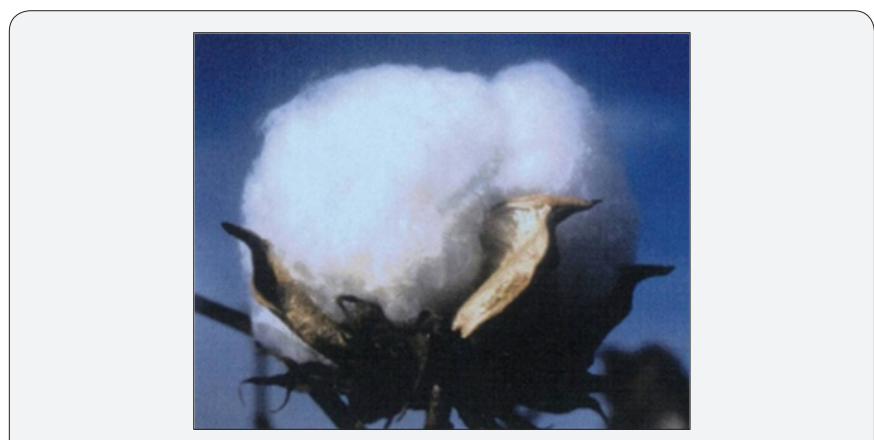

Figure 1: Cotton fibre.

Table 1: Textile Fibres.

\begin{tabular}{|c|c|c|c|}
\hline & Categories & Sub- Categories & Examples \\
\hline \multirow{3}{*}{ Natural Fibres } & \multirow{2}{*}{ Organic } & Vegetable Fibres & Cotton Flax, Hemp, Jute Sisal \\
\hline & & Animal Fibres & Wool Silk \\
\hline & Inorganic & Mineral Fibres & Asbestos Basalt \\
\hline \multirow{4}{*}{ Man-made Fibres } & \multirow{3}{*}{ Organic } & $\begin{array}{c}\text { Regenerated Natural Polymer } \\
\text { Fibres }\end{array}$ & Regenerated cellulose fibres Viscose Cupro \\
\hline & & Modified Natural Polymer Fibres & $\begin{array}{l}\text { Modified cellulose fibresCellulose acetate } \\
\text { Cellulose triacetate }\end{array}$ \\
\hline & & Synthetic Polymer Fibres & Polyamide Polyester Polyolefin Polyacrylic \\
\hline & Inorganic & Synthetic Non-polymer Fibres & Glass Carbon \\
\hline
\end{tabular}




\section{Fibre manufacturing/preparation}

Natural fibres, known as staple fibres when harvested, include vegetable fibres (e.g. cotton) and animal fibres (e.g. silk and wool). Staple fibres may be converted to yarn through a series of manufacturing operations, including ginning, opening, blending, carding, combing, drafting, etc, all of which are a part of the staple-fibre spinning process.

Natural mineral fibres including basalt fibres (both continuous filament and staple fibres) and asbestos (a staple fibre) are known.

Manmade fibres include natural-polymer regenerated organic fibres, including regenerated cellulose (viscose, cupro, polynosic and lyocell) and modified-cellulose fibres, including cellulose acetate and triacetate, both types being manufactured from wood fibres or other natural sources of cellulose. The regenerated fibres are chemically the same as the original natural polymer from which they are made while the modified fibres are chemically different from the parent polymer [2].

Manmade fibres also include another important and widely useful category of fibres, the synthetic organic fibres, which are made from chemical raw materials originating from the petrochemical industry. Outstanding examples of synthetic manmade fibres are polyamide, polyester, polyolefin and polyacrylic fibres.

Manmade fibres are produced by converting fibre-forming polymer by extrusion of polymer melt or solution through fine holes into one of two basic forms: filament yarn or staple-length (short-length) fibres; the latter form is to facilitate spinning into 'staple-spun yarn' as stated above for the natural fibres.

Manmade inorganic fibres include glass and carbon fibres, which are made as continuous filaments or as staple fibres. The staple fibres of both of these materials are used in felts and composites. The continuous filament of glass is processed by spinning, twisting, and sizing. The continuous filaments of carbon are produced by very high temperature heat-treatment processes [3].

\section{Yarn manufacturing}

Staple (including natural and manmade) fibres are transformed into yarn through grouping and twisting operations. Other fibres are processed using extrusion spinning operations. Yarn manufacturing typically includes the following operations:

o Vegetable fibres: ginning, fibre blend, carding, combing, spinning (ring-spinning and open-end spinning) and twisting

o Animal fibres: scouring, fibre blend, carding, combing, spinning (ring) and twisting

o Mineral fibres: spinning, twisting and sizing

o Regenerated natural-polymer fibres: carding, combing, spinning (ring) and twisting o Manmade fibres: carding, combing, spinning (ring or open end) and twisting (Figure 2).

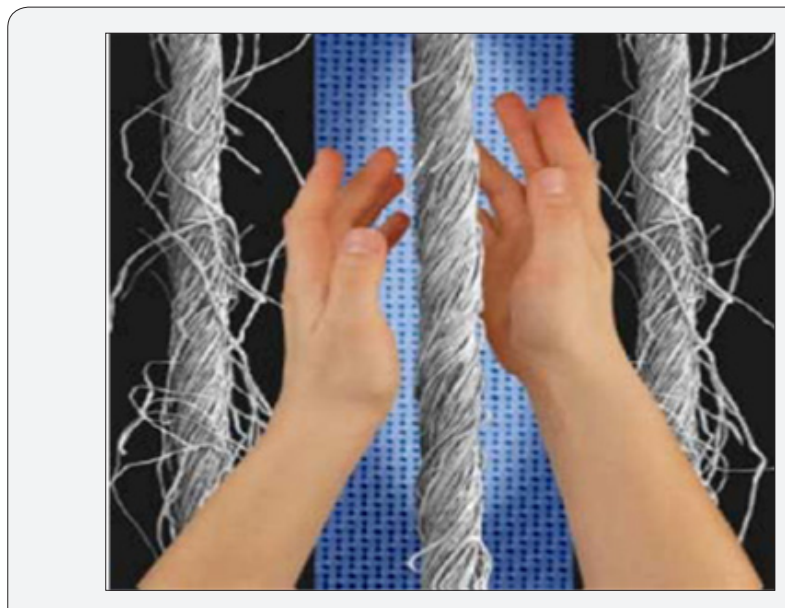

Figure 2 : The twist in staple-fibre yarn; the fuzzy surface is due to protruding loose fibre ends.

\section{Continuous filament yarn may be used directly or after the following operations:}

o Natural fibres (e.g. silk): twisting

o Regenerated natural-polymer fibres: spinning and twisting

o Manmade fibres: spinning, twisting and texturising

o Inorganic fibres: spinning (glass) or pyrolysis (carbon), twisting and sizing

o Continuous filament yarns are normally smooth and lustrous due to absence of 'fuzz' (Figure 3).

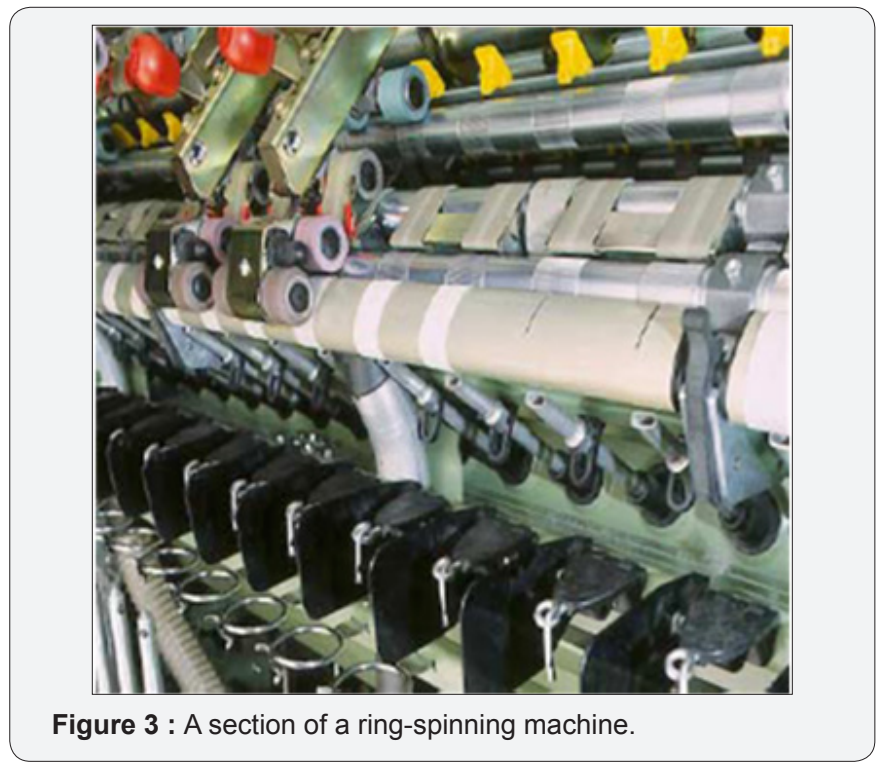

\section{Fabric Production}

Winding: Winding involves transferring yarn from one type of package to another, usually a smaller to a larger one, to facilitate subsequent processing [4]. Precision winders are 
used primarily for filament yarn and produce packages with a diamond-patterned wind. Drum winders are used mainly for spun yarns (Figure 4).

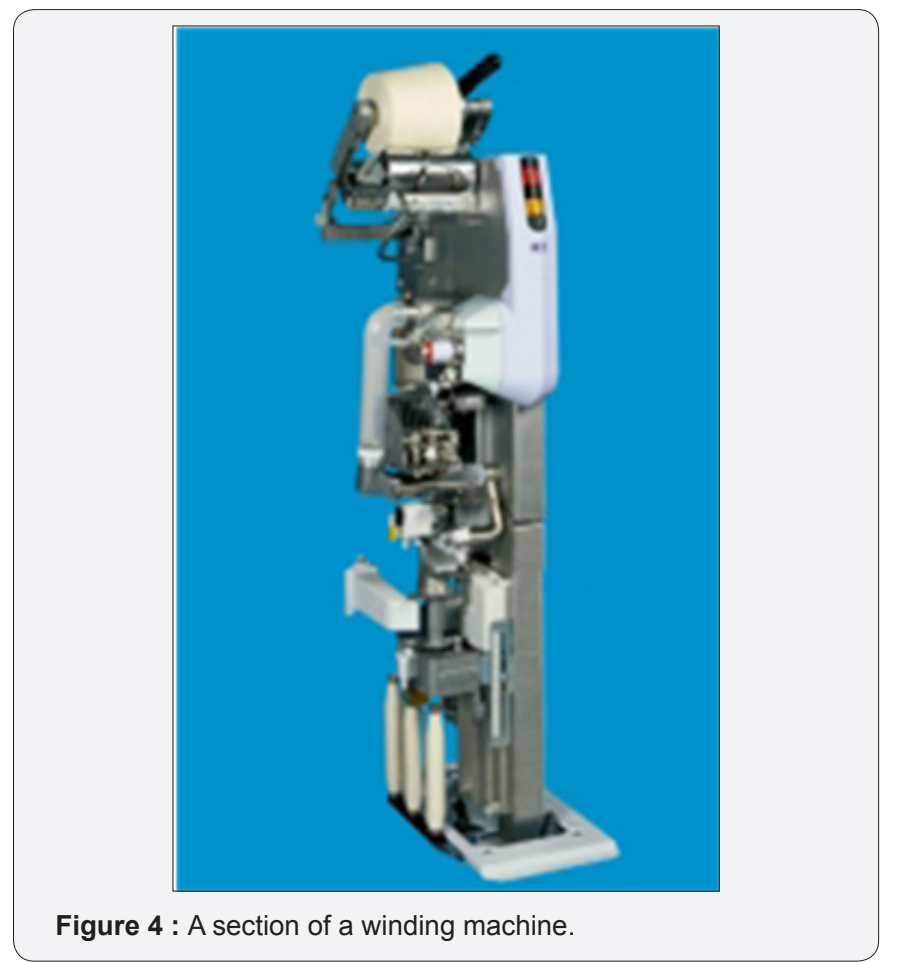

Warping: Warping involves winding a portion of the total number of ends of a warp in full width onto a back beam.

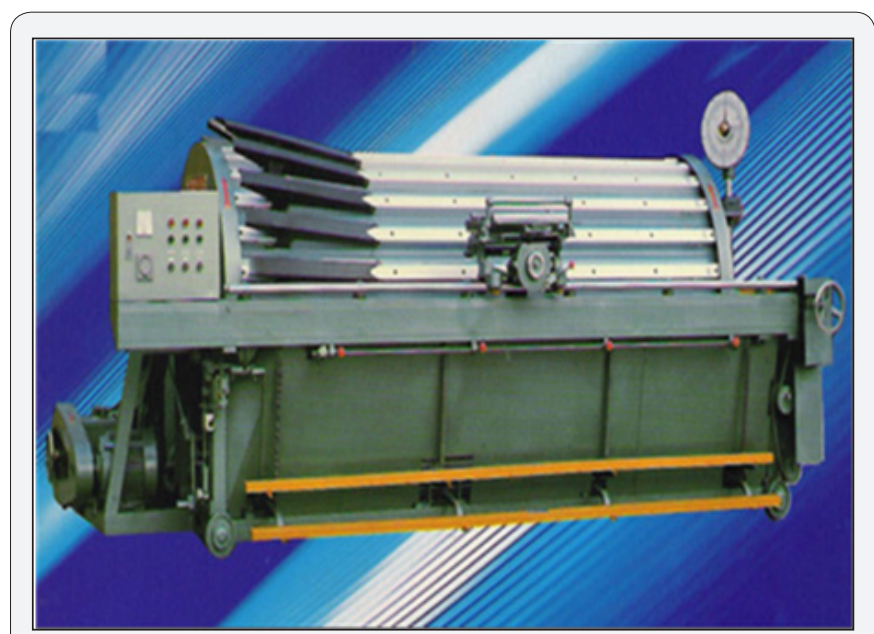

Figure 5 : A sectional warping machine.

Section Warping: Section warping is a machine method of preparing a warp sheet on a beam. The process involves winding warp threads in sections on a reel and beaming off the complete warp from the reel onto a warp beam (Figure 5).

\section{Beaming}

Beaming is an activity during warp-making in which ends, withdrawn from a warping creel, are wound onto a beam to a length that is a multiple of the loom warp length. Several similar beams (known as a set of back beams) provide the total number of ends required in the woven warps. Beaming is usually implemented for bulk production of grey warp. Direct beaming is a specific single-stage method.

\section{Sizing}

Sizing involves applying special compounds and chemicals to warp yarn to bind the surface fibre together and protect the yarn against abrasion during weaving. The primary sizing compounds include starch, gelatin, oil, wax and manufactured polymers (such as polyvinyl alcohol, polystyrene, polyacrylic acid and polyacetates) (Figure 6).

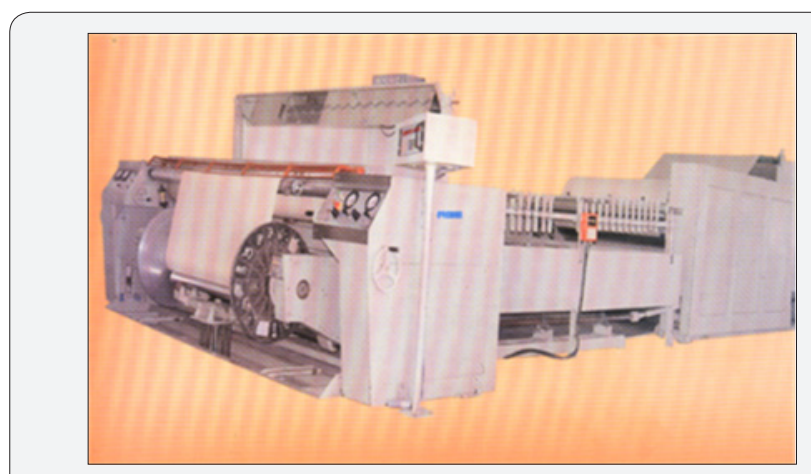

Figure 6 : Sizing machine.

The beam-to-beam sizing method transfers a warp from a warp beam to a loom beam, while it is being sized. The sized warp is dried by hot air or by contact with steam-heated cylinders during its transfer to the loom beam.

\section{Fabric manufacture}

The most important methods for fabric manufacture are weaving and knitting. Weaving is done using looms, which constitute a combination of devices and techniques that permit a warp sheet to be tensioned and a shed to be formed with the aid of heddles. There are many kinds of looms including shuttle, projectile, rapier and fluid jet. The shuttle loom uses a weft insertion device that propels the weft yarn across (over and under) the warp yarns [5].

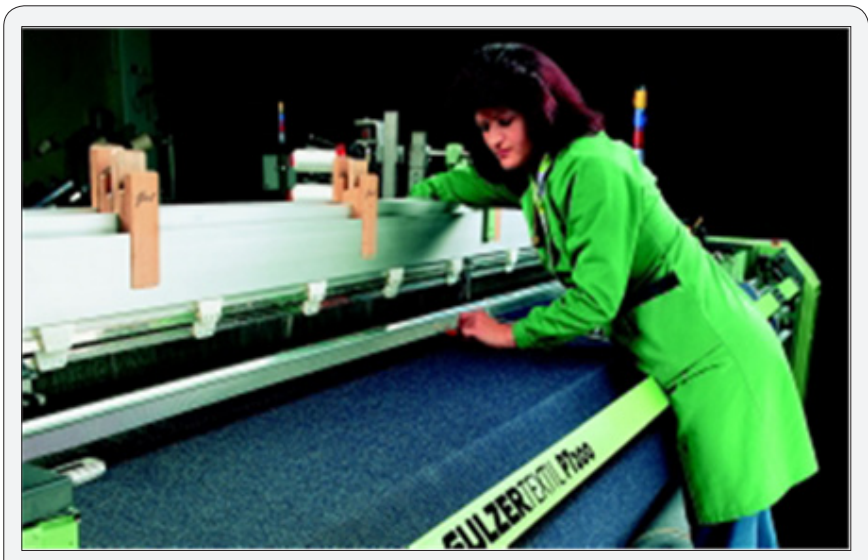

Figure 7 : A projectile loom. 
The projectile loom uses a projectile that conveys the weft through the shed and leaves a trail of yarn behind it. The rapiertype loom conveys a weft yarn from a stationary package through the shed. Rapier looms are simpler and more versatile than dummy-shuttle looms; however their weaving speed is slower. The two types of fluid-jet looms are air jet and water jet (Figure 7).

Knitting is a method of converting yarn into fabric by intermeshing loops, which are formed with the help of needles. Two weft-knitting technologies are used and these include flatbed knitting (used for heavier gauge material) and circular knitting, as in the adjacent figure. Warp-knitting technologies include chain (e.g. lace, light tricot), Raschel (e.g. lace, velvet, technical fabrics) and crochet (e.g. technical fabrics).

Tufting is a process used to make carpets. As neither weaving nor knitting is involved, tufted carpets are 'non-woven' textiles. These fabrics are produced by means of mechanical-bonding, water-bonding and air-bonding machines. Braiding is an interlacing technology in which two sets of continuous yarns are interwoven symmetrically around an axis, the resulting product being a continuous strand, thick as a cord (Figure 8).

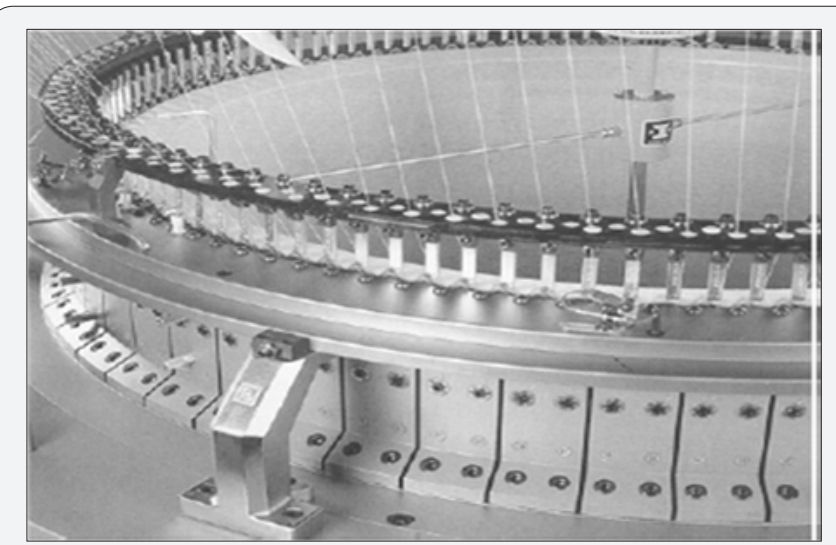

Figure 8 : A section of a weft-knitting machine showing the circular array needles.

\section{Finishing processes}

Woven and knit fabrics are not normally processed into apparel or other finished goods until the undyed and unfinished fabrics, known as grey or greige fabrics, have passed through several chemical processes, which are predominantly wetprocessing stages. These processes convert them into 'finished' fabrics, enhancing their aesthetic appearance, durability and serviceability. Wet processing or finishing processes include the main processes of fabric preparation, namely dyeing, printing, and other specific treatments. These phases treat fabrics with chemical and aqueous or other liquor baths and often require several washing, rinsing and drying steps.

\section{Preparation}

Preparation (also known as pre-treatment) of textile fabric for dyeing, printing or other finishing process consists of a series of chemical treatment and washing steps. The efficiency with which these processes are performed determines the results of subsequent textile finishing processes. Processing chemicals are usually used to remove natural impurities and these may interfere with dyeing, printing and finishing if not properly removed. Typical preparation treatments include desizing, scouring and bleaching; other processes such as singeing (for fabric) or gassing (for yarns) and mercerizing are often also used. All of these pre-treatment steps are intended to alter the textile material either chemically or physically. Solid, liquid or gaseous pollutants may be generated from many of these processes, the most important of these being metals, organic compounds and phosphorus present in the surfactants and detergents.

\section{Desizing}

Desizing is the preparation step used to remove size materials applied before weaving in the sizing operation. Size is a necessary reinforcement for the warp threads during the tension-ridden weaving process. However, once the fabric is made, it constitutes an added impurity that prevents fabric wetting and its interaction with chemicals, and thus has to be removed. With regard to natural fibres, the size materials are most often water-insoluble starches or mixtures of starch and other materials. In the desizing step, the use of enzymes capable of breaking starches into water-soluble sugars is nowadays more prevalent than traditional methods that use bacterial action or mineral acids. The sugars are then removed by rinsing treatments before subsequent steps. Manmade fibres, on the other hand, are generally sized with water-soluble sizes that facilitate easy removal by means of hot-water treatments.

\section{Singeing}

Singeing involves the burning away of protruding fibres from fabric or yarn by passing it rapidly across a gas-ignited flame or over heated copper plates. In the case of yarn the term gassing is often used instead of singeing. Sometimes singeing precedes the desizing step, the desizing liquor acting as a quenching medium for the hot, singed fabric.

\section{Spinning}

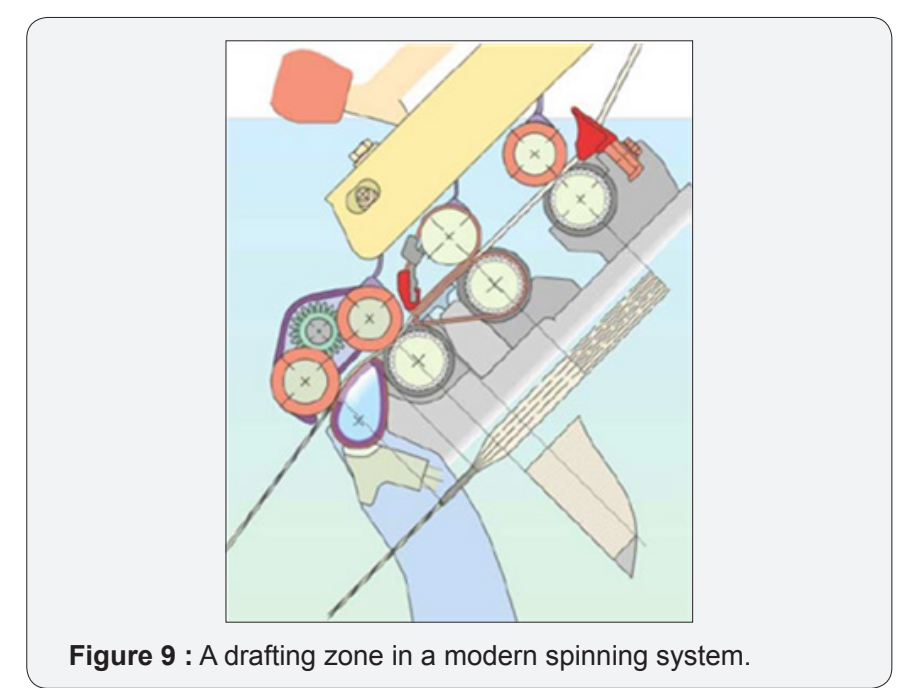


When staple fibre is the raw material, spinning involves the conversion of the fibre into yarn through process steps that are designed to free the fibre of adventitious dirt, align them in parallel array to form long continuous strands, drawing them to become finer (drafting) and finally twisting them to give a yarn. The last step is performed traditionally on a ring-spinning machine (Figure 9).

As of today, the ring-spinning technique has gone through modifications, the most important of these being referred to as compact or condensed spinning, an improvement that reduces fibre waste production while producing yarn of greater strength, enhanced appearance and a lower degree of hairiness (i.e., less fuzzy).

\section{Mercerising}

Mercerising is actually a finishing process that is often included along with pre-treatment processes. It is used mainly for cellulosic textile fibres (yarn and fabric) and involves treating the material with a concentrated solution of caustic alkali, usually sodium hydroxide. The treatment causes the fibres to swell and increases the strength and dye affinity of the mercerised material. More recently, the effects of mercerisation have been more or less brought about by means of a low-temperature liquid ammonia treatment, originally called the 'Prograde' process.

\section{Bleaching}

Bleaching is a process to improve the whiteness of textile material. Commonly used chlorine-based bleaches include sodium hypochlorite and sodium chlorite, the former mostly for cellulose textiles and the latter mainly for the synthetic fibres. Hydrogen peroxide is regarded as a universal bleaching agent due to the fact that it can be used for almost all fibres. Peracetic acid bleaching is sometimes used for some synthetic fibres like nylon that cannot be bleached by means of hydrogen peroxide.

\section{Dyeing}

Dyeing is a process of coloration of textile material, which can be dyed in several forms. For instance, it can be dyed as loose stock (loose fibre), as a continuous strand formed during an intermediate stage in spinning (wool sliver, referred to commonly as 'top'), as yarn package (cop, cone, cheese, beam), as yarn in skein form (hank-dyeing), as woven or knitted fabric (piece dyeing), etc.

Dyeing machines are available to dye textiles specifically in any of the above forms. Modified and improved versions of dyeing techniques are also available for some forms of textile material. For example, fabric may be dyed in a jigger, winch or jet-dyeing machine, depending on the fabric type. The machines have two basic variants, one in which the textile to be dyed is stationary while the dye liquor is mechanically circulated (e.g. yarn package, hank-dyeing, beam dyeing, etc) and the other in which the textile material is run by mechanical means through dye liquor that is virtually static (jigger, winch, etc.). The jetdyeing technique is a relatively recent improvement of the winch principle in which both the liquor and the fabric are caused to move. This modification produces good dyeing results while bringing about savings in time and process cost. A soft-jet dyeing machine is also available for delicate fabric like knitted fabric that is susceptible to structural distortion in a conventional jet machine (Figure 10).

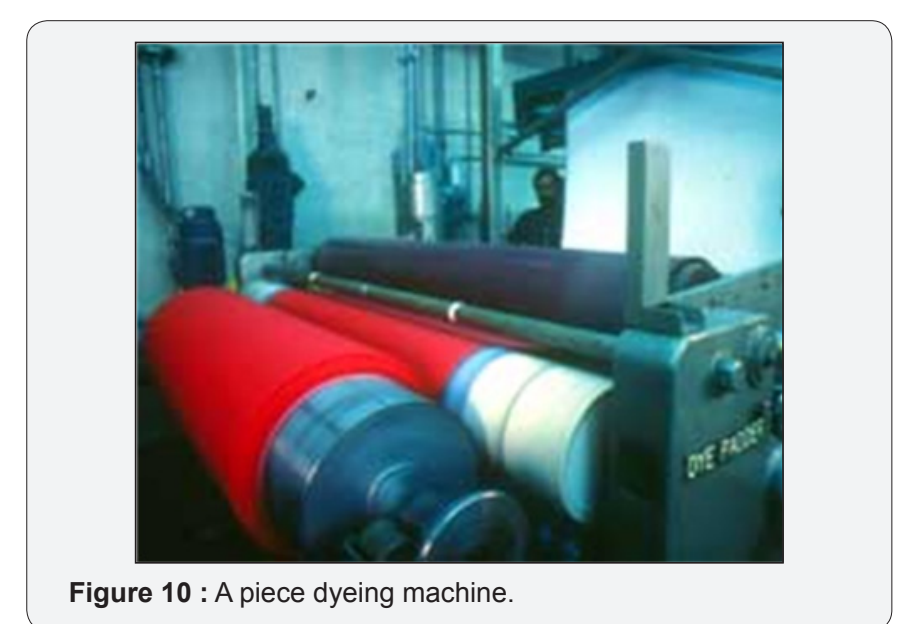

Dyeing is conducted in a textile manufacturing plant or in specialty dye-houses. Textiles are dyed using a wide range of chemicals and dyestuffs [6].

Dyes are generally synthetic chemical products that are marketed in various forms like powder, granular, paste and liquid dispersion. Textile material may be dyed in batches of specific sizes, or in a semi-continuous manner or even in a continuous manner on 'continuous dyeing ranges'.

In batch dyeing, a specific quantity of the textile material is loaded into a dyeing machine and kept in continuous contact with the dye liquor, either by keeping the textile material in continuous motion or by circulating the liquor. Dyeing assistants (special chemicals) are included in the dye bath and/or specific conditions of dyeing are maintained to obtain efficient dyeing results. The dye is then caused to attach itself in one of many ways to the fibre (i.e. it is fixed) in a process that uses chemicals and/or heat. The 'fixing' treatment will still leave a small amount of the dye on the fibre surface. A hot wash in the presence of soap will normally remove the unfixed dyes and chemicals from the textile material.

The Material-to-Liquor Ratio (MLR) i.e. the ratio of the mass of the textile material to that of the dye liquor used in the dyeing equipment plays a significant role in the economics and the efficacy of dyeing in the case of batch dyeing. Winches use an MLR of 1:40, while for jiggers it is around 1:5. The soft-jet machines which are an innovative modification of the winch design use MLRs in the range 1:10/15. The MLR for package dyeing machines is around 1:10 and for hank-dyeing machines around 1: 25/30. The higher the MLR, the higher will be the dyes and chemicals used and higher too the energy requirements and the wastewater discharge. There has been a conspicuous move towards low MLR dyeing machines in the recent past [7]. 
As opposed to batch dyeing, continuous dyeing processes take the textile material, in one continuous run through a 'dyeing range', where the steps of dye application, fixation and washingoff are carried out sequentially without any break in process. Ready-to-dye fabric is fed in at one end and the dyed and washed fabric emerges at the other end. Much of the polyester and cotton-polyester blended textiles are dyed in a continuous range.

\section{Printing}

While dyeing colours the whole of the textile material, printing produces designs or motifs on the fabric by applying a colorant on discrete parts of the textile. Several printing techniques are prevalent in current textile practice, e.g. roller printing, flat-screen printing, rotary-screen printing, sublimation printing and ink-jet printing.

In roller printing, design-engraved metal rollers press colour paste on to the fabric to be printed in a continuous manner. In screen printing, a screen which has the design or motif to be printed as a fine mesh is kept pressed against the textile to be printed while the colour paste is forced through to produce a printed fabric. In both of these processes, there are as many engraved rollers or screens as there are colours in the designs. Transfer printing is a relatively recent technique in which design from a printed paper is transferred to fabric in close contact with it by means of heat and pressure in a continuous manner. Transfer printing is predominantly used in the printing of synthetics, especially polyester (Figure 11). Traditional methods of printing like block or stencil printing are practiced in the small-scale production of cotton printed textiles. Printed material is always dried and then steamed or subjected to dry heat (i.e. cured) to 'fix' the prints.

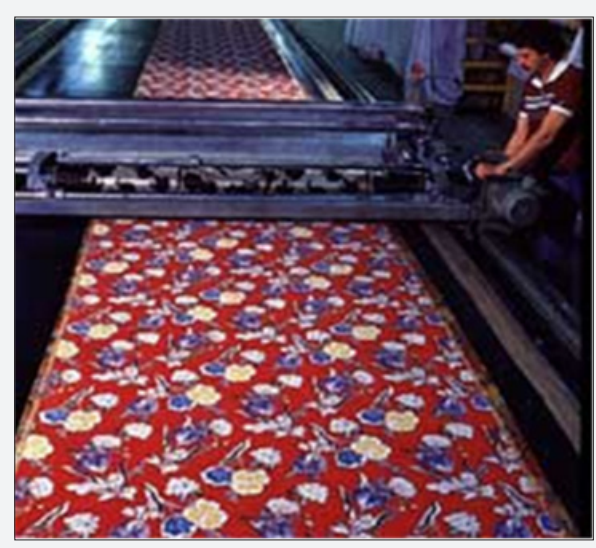

Figure 11 : Screen printing.

\section{Stentering}

Stentering is a mechanical process that stretches wet fabric widthways and dries it by means of hot air. The result is a flat, creaseless fabric of desired specific width.

\section{Coating}

These processes are used mainly for the production of industrial or 'technical' textiles. Coating involves the application of a chemical in paste form on one or both sides of a textile material. Drying and curing of the coated textile are necessary to allow the coating material to form a bond with the fabric. Several techniques of coating are used.

Direct coating involves uniform spreading of the coating on a textile material with a knife. In roller coating, the coating is applied to a running textile material by means of a roller. In transfer coating the coating is transferred from a temporary substrate to the textile material and fixed on to it by means of adhesive bonding [8].

\section{Calendering}

Most textile material is subjected to a calendaring finish to add an attractive sheen to the final product. The textile fabric is passed successively between hard and soft rollers in contact with each other to end up with and 'ironed' effect. Different calendared effects are possible by varying the number, type of roller surface and the relative speeds of successive rollers. However, these finishes are only temporary in nature and wear off during the initial stages of life of the textile.

\section{Specific necessities in wet processing}

Wet processing produces the most significant amounts of effluent and waste in textile operations. Additionally, it requires significant quantities of energy for heating and cooling baths and for drying the processes textiles. The specific ways that are used to heat up or cool process liquor vary considerably depending on final products and applications, textile chemical processing practices and the type of fibre involved. Synthetic fibres are generally easier to process than the natural fibres, which often require a greater number of processing steps. Variation in the wet processes used also depend to a greater or lesser extent on the final properties desired of the end product, such as tensile strength, flexibility, uniformity, lustre and other functional properties.

\section{Embellishment of end products}

The increasing demand for aesthetic appearance and uniqueness has brought about a sea change in the way textiles are made and finished today. Manufacturing of textile end products includes finished fabric decoration, such as embroidery, garment assembly, home textiles and other industrial uses of finished textiles. Embroidery is the art of surface ornamentation of fabric or other materials with designs and motifs composed of special stitches using embroidery thread or yarn and a needle. Embroidery also involves the use of other materials such as metal strips, pearls, semi-precious stones, colourful metallic pieces and so on.

Garment production is in general a highly labour-intensive activity that makes use of a variety of sewing machinery for a host of stitches and seams that help in the construction of men's, women's and children's wear. 


\section{Conclusion}

The importance of textiles as an inevitable contributor to the growth of civilisation is indisputable and can never be overemphasised. Textiles are of interest to people in all walks of life, irrespective of any kind of distinction. This is probably one of the reasons for the incredible growth and expansion of textiles today and the amount of innovation that it has generated. The result is a vast variety of textile materials catering to a wide range of applications. Further developments can be anticipated, perhaps at a rate greater than ever before.

\section{References}

1. Mishra SP (2000) Fibre Science and Technology, New Age International Publishers, New Delhi, India.
2. Chattopadhyay R (2002) Advances in Technology of Yarn Production, NCUTE, IIT Delhi, India.

3. Lord PR (2002) Yarn Production: Science, Technology and Economics, The Textile Institute, Manchester, USA.

4. Murphy WS (2001) Hand Book of Weaving. Abhishek Publications, Chandigarh, India.

5. Murphy WS (2000) Textile Weaving \& Design, Abhishek Publications, Chandigarh, India.

6. Saville BP (1999) Physical Testing of Textiles, The Textile Institute, Woodhead Publishing Limited, Cambridge, USA.

7. Anbumani N (2006) Knitting Fundamentals, Machines, Structures and Developments, New Age Publication, New Delhi, India.

8. Khatwani PA (1999) Weaving - II Shuttleless Looms, NCUTE Publication, Delhi, India.

\begin{tabular}{|l|}
\hline \multicolumn{1}{|c|}{ Your next submission with Juniper Publishers } \\
will reach you the below assets \\
- Quality Editorial service \\
- Swift Peer Review \\
- Reprints availability \\
- E-prints Service \\
- Manuscript Podcast for convenient understanding \\
- Global attainment for your research \\
- Manuscript accessibility in different formats \\
( Pdf, E-pub, Full Text, Audio) \\
- Unceasing customer service \\
Track the below URL for one-step submission \\
https://juniperpublishers.com/online-submission.php \\
\hline
\end{tabular}

